\title{
A VOXEL-BASED TECHNIQUE TO ESTIMATE THE VOLUME OF TREES FROM TERRESTRIAL LASER SCANNER DATA
}

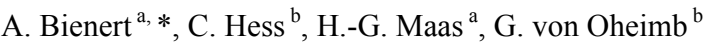 \\ ${ }^{a}$ Technische Universität Dresden, Institute of Photogrammetry and Remote Sensing, 01062 Dresden, Germany \\ (anne.bienert, hans-gerd.maas)@tu-dresden.de \\ ${ }^{\mathrm{b}}$ Leuphana Universität Lüneburg, Institute of Ecology, 21335 Lüneburg, Germany \\ (hess, vonoheimb)@leuphana.de
}

Commission V, WG V/5

KEY WORDS: Terrestrial Laser Scanning, Point Cloud, Tree, Volume Estimation, Voxel

\begin{abstract}
:
The precise determination of the volume of standing trees is very important for ecological and economical considerations in forestry. If terrestrial laser scanner data are available, a simple approach for volume determination is given by allocating points into a voxel structure and subsequently counting the filled voxels. Generally, this method will overestimate the volume. The paper presents an improved algorithm to estimate the wood volume of trees using a voxel-based method which will correct for the overestimation. After voxel space transformation, each voxel which contains points is reduced to the volume of its surrounding bounding box. In a next step, occluded (inner stem) voxels are identified by a neighbourhood analysis sweeping in the $X$ and $Y$ direction of each filled voxel. Finally, the wood volume of the tree is composed by the sum of the bounding box volumes of the outer voxels and the volume of all occluded inner voxels. Scan data sets from several young Norway maple trees (Acer platanoides) were used to analyse the algorithm. Therefore, the scanned trees as well as their representing point clouds were separated in different components (stem, branches) to make a meaningful comparison. Two reference measurements were performed for validation: A direct wood volume measurement by placing the tree components into a water tank, and a frustum calculation of small trunk segments by measuring the radii along the trunk. Overall, the results show slightly underestimated volumes $(-0.3 \%$ for a probe of 13 trees $)$ with a RMSE of $11.6 \%$ for the individual tree volume calculated with the new approach.
\end{abstract}

\section{INTRODUCTION}

The trunk is the economically most important part of a forest tree, and the determination of the stem volume is a main task in forestry. Besides the value of the standing timber to predict the harvesting profit, the volume (or its temporal change) may also give information about the success of silvicultural methods. A very accurate direct measurement of the trunk volume can be done with xylometry methods (i.e. water displacement methods). However, this results in a time consuming process and a destroyed tree. Alternatively, non-destructive methods preserve the stand structure, but at the expense of the accuracy of volume determination. Usually, these methods are based on prediction models making use of different stem diameters measured along the trunk. A simple volume calculation of the tree trunk is achieved by adding up the volumes of different rotationally symmetrical bodies along the stem axes. Beyond this, terrestrial laser scanning offers an efficient, accurate and non-destructive way to estimate tree volume of standing trees. Terrestrial laser scanning is a fast and efficient tool to capture 3D-information of complex object structures. The interest on terrestrial laser scanning for the use in forestry applications has increased in recent years. A common field of application for terrestrial laser scanners is the determination of forest inventory parameters (e.g. Hopkinson et al., 2004; Thies \& Spiecker 2004; Watt et al., 2005; Maas et al., 2008). The two important tree parameters, tree height $(h)$ and stem diameter at breast height $(d b h)$, are further used in prediction models to estimate the volume or biomass of a tree in a non-destructive way (e.g. Kankare et al., 2012). Dassot et al. (2012) calculate the tree volume by modelling the stem and branch structure with fitted cylinders. Biomass can also be estimated by analyzing data in a voxel space structure (e.g. Moskal \& Zheng, 2011; Hosoi et al., 2013). Hosoi et al. (2013) use multiple-scans and obtain an averaged error of $6.8 \%$ of the volume. A simple approach is summing up all filled voxels after allocating points into a voxel structure. Obviously, the estimated wood volume depends on the size of the utilized voxels. On the one hand, a large voxel size will lead to a large overestimation of the tree volume (Figure 7). On the other hand, an underestimation is given if the voxel size falls below the stem or branch diameter and empty voxels located inside the stem or branch are missed. The later may be compensated by filling those empty voxels by 3Dmathematical morphology operations. Moskal \& Zheng (2011) use a voxel-based method to calculate the tree volume by summing up the filled voxels and adding an adjustment factor, which represents the trunk volume calculated by $h$ and $d b h$, obtained from the point cloud. The authors present volumes derived from single-scans, which match only $18 \%$ of the total tree volume because of scan shadows. Regarding the stem volume, diameter profiles along a tree stem can also be used to model conical frustums (Pueschel et al., 2013).

The goal of this paper is the development of a novel voxelbased algorithm to precisely determine the wood volume of standing trees. The quality of the results of the presented method should be comparable to those of conventional

\footnotetext{
* Corresponding author.
} 
methods. The outline is as follows: section 2 introduces the data sets used. Afterwards, section 3 presents the new-developed voxel-based algorithm for volume determination. Extensive tests on different point clouds are presented in section 4 . Results from processing a total of 13 trees will be presented and will be compared to volume values obtained by the water displacement method, which was applied after harvesting the trees. Finally, a discussion will be given, and a summary closes the article.

\section{DATA SETS}

All trees were scanned on an almost windless day in May 2013 under leaf-off conditions with a FARO Photon 120 laser scanner. The trees were scanned with an horizontal and vertical angular step size of $0.036^{\circ}$, which leads in a point spacing of $2.5 \mathrm{~mm}$ at $4 \mathrm{~m}$ distance. Up to 244,000 points per second were measured, and the scanner had a clear view without occlusions to the trees and a mean distance between $3 \mathrm{~m}$ and $4 \mathrm{~m}$. Consequently, the point clouds have $10,000 \leq n \leq 200,000$ points, depending on the tree size and branch structure. After scanning, all point clouds were filtered with the filter tool Statistical Outliers Remover of the open-source point cloud editing software CloudCompare to reduce typical stray points.
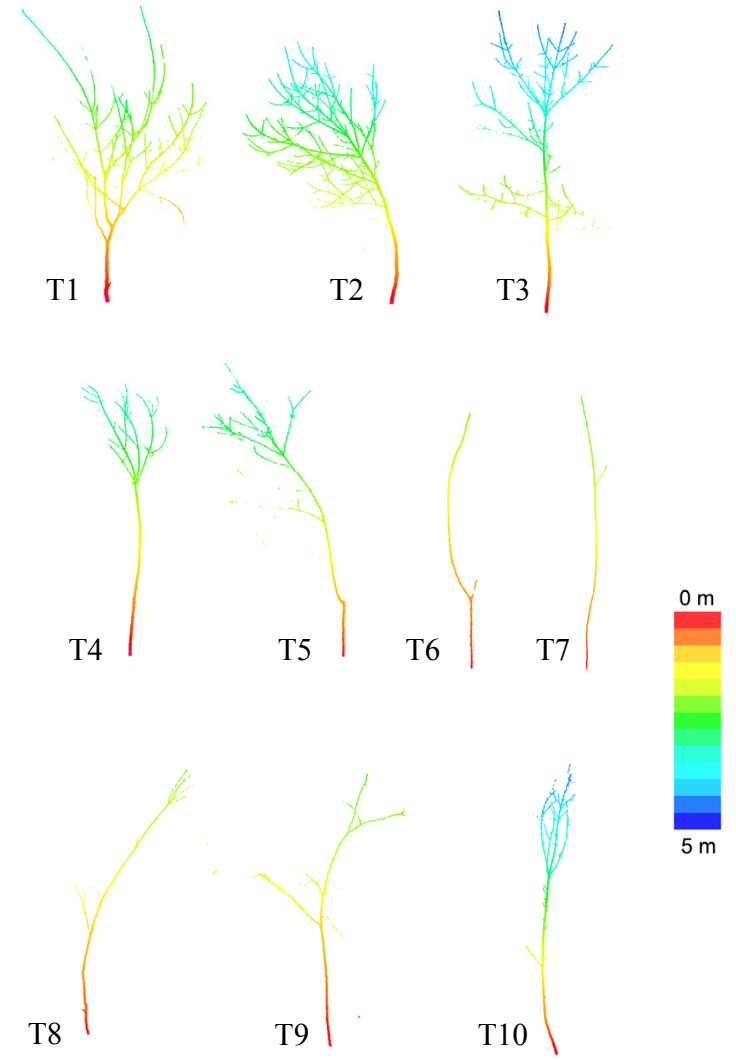

T11
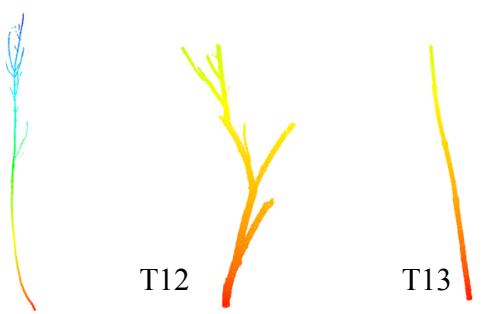

Figure 1. Tree point clouds of data set A.
In this study, the scanned trees are young trees $(h<5)$ with a very fine structure, motivated by research in young mixedspecies tree plantations of the BEF-China project (Li et al., 2014).

Data set A is a sample set of 13 young Norway maple (Acer platanoides) trees, each one scanned from four nearly rectangular scanner positions. Afterwards, the trees were destructively harvested for a direct volume analysis. Furthermore, all scanned trees as well as their representing point clouds were separated in different components (stem, branches) to make a meaningful comparison. The point clouds were separated interactively using the software CloudCompare, to ensure the same size to the cut components. Data set $\mathrm{B}$ is a subset of two trees (Figure 1 - T2 and T6) of data set A, which were scanned twice from almost the same viewing directions for a repeatability analysis.

\section{METHODS}

The processing scheme consists of three processing steps. A similar algorithm was presented by Hosoi et al. (2013) to estimate the wood volume by considering the empty space inside a point contour. For an optimized result, the algorithm is applied to a filtered tree point cloud without stray points (as they frequently occur in the data of certain scanners). If a leafoff scanning cannot be performed, the leaves may be filtered out analysing the point density and distribution inside a medium scale voxel size, making use of the fact that the voxels including stem and large branches are characterized by a high point density and a spatial arrangement which describes a curved area

\subsection{Voxel-based algorithm}

\subsubsection{Bounding box generation}

At first, a voxel space with a voxel size $s_{\text {voxel }}$ smaller than the maximum stem diameter is calculated using the method outlined by Bienert et al. (2010). All points are allocated to an individual voxel $c_{i}$. As one can see in Figure 2a, the use of the voxel volume of the outer hull will lead to an overestimation, caused by volumes of voxels only partly filled with points. Therefore the voxel volume has to be adjusted to the actual point distribution. For that purpose, an axis-parallel bounding box is determined for each voxel (Figure 2b), given that enough points are included and a $3 \mathrm{D}$-volume is spanned. This step is crucial to adjust the volume to the space which is actually filled.
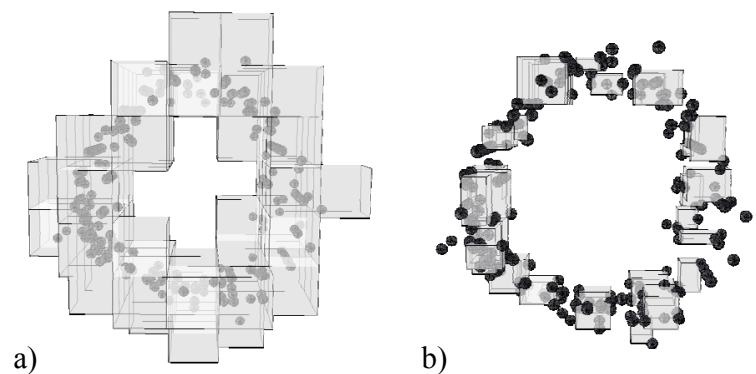

b)

Figure 2. a) Voxel space with filled voxels containing at least one point; b) Axis-parallel bounding boxes of the filled voxels. 


\subsubsection{Identification of occluded voxels}

The second step addresses the identification of the occluded (inner) voxels (Figure 4a). These occluded cells are identified by sweeping in horizontal layers $X$ - and $Y$-direction of each filled voxel, similar to the method of Hosoi et al. (2013). Starting with the first filled voxel $c_{i}(X, Y, Z)$, the $Y$-direction is analysed, running through the complete range $\left(Y_{\min } \leq Y \leq Y_{\max }\right)$. Passing an empty voxel increases its counter $l$ inside the column (yellow voxels in Figure 3a). Afterwards, the $X$-direction for the remaining filled voxels is checked. The same procedure is repeated for filled voxels in the $X$-direction (Figure $3 b$ ). Hereafter the next horizontal layer is processed.

Those voxels that have been labelled twice and have a counter $l=2$ (orange voxels in Figure $3 \mathrm{c}$ ) are regarded as potentially occluded voxels. A second check is performed, analysing the neighbourhood of the potential candidates (Figure 3d). Inside a preset radius $r$, which depends on the largest stem radius of the tree, the neighbouring voxels are checked. Starting with a voxel candidate the $X$ - and $Y$-direction is checked. The cell is occluded if the neighbouring cells are labelled with the counter $l=2$ and/or a filled voxel is noted in each direction. If one empty voxel $(l=0$ or $l=1)$ is recognized, the candidate is not occluded. In comparison to a classical 3D mathematical morphology, this approach is insensitive for grouped or single voxels next to the stem contour (due to branches or noisy points).

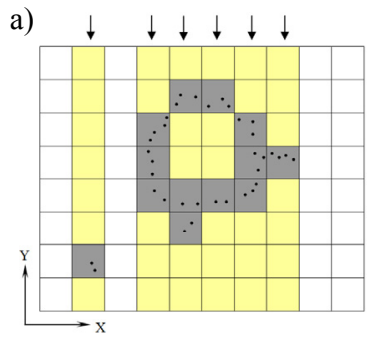

b)

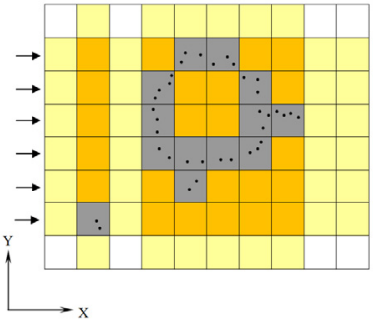

d)

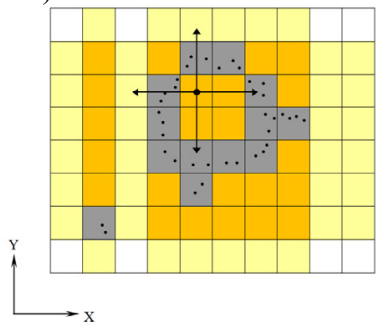

c)

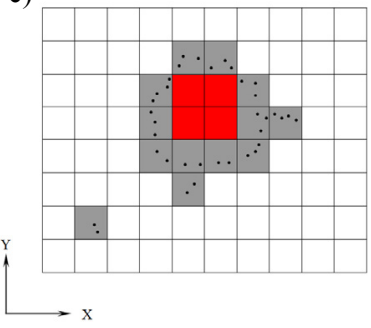

Figure 3. Identification of the occluded voxels: a) Scanning in $Y$-direction; b) Scanning in $X$-direction; c) Checking of the candidates; d) Occluded voxels.

\subsubsection{Bounding box expansion}

Finally, the bounding boxes have to be expanded to the sideface of a recognized neighbouring shadowed or filled voxel, as the bounding box method will lead to gaps between bounding boxes in neighbouring voxels. By checking the nearest neighbours of all filled voxels (white voxels in Figure 4a), the sides to the adjacent filled or shadowed neighbor voxels are identified. As soon as a filled or occluded voxel is recognized, the size of the bounding box is enlarged to the side-face. Thus, the bounding boxes join each other without empty space between the cells.
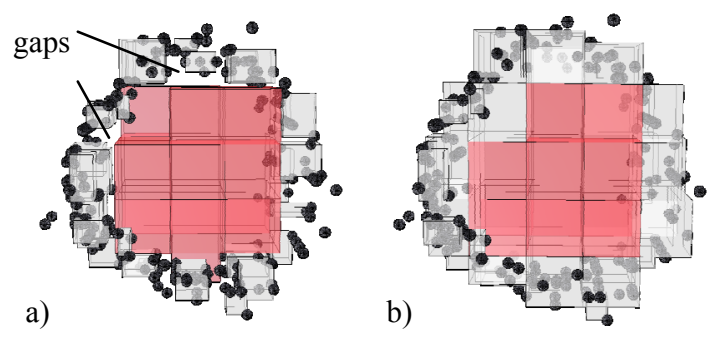

Figure 4. a) Bounding boxes with gaps and occluded voxels; b) Expanded bounding boxes with occluded voxels (red).

\subsection{Volume estimation via voxels}

3.2.1 Voxel volume method $V_{V o x}$

As mentioned above, the simplest way to estimate a tree volume is adding up the volume of all filled voxels, independent on the number of points therein. The determined volume $V_{V o x}$ (Eq. 1) depends on the utilized voxel size $s_{\text {voxel }}$. Generally, an overestimation with the volume has to be assumed.

$$
V_{V o x}=n_{f} \cdot\left(s_{\text {voxel }}^{3}\right)
$$

where:

$$
\begin{array}{ll}
n_{f} & \quad \ldots \text { number of filled voxels } \\
s_{\text {voxel }} \quad \ldots \text { unit voxel volume }
\end{array}
$$

3.2.2 Bounding box and occluded voxel volume $V_{\text {total }}$ The total volume $V_{\text {total }}$ represents the wood volume of the tree (Eq. 2). It composes the sum of all bounding box volumes $V_{B B}$ of the filled voxels (Eq. 2a, 2b) and the sum of all occluded voxel volumes $V_{\text {Shadow }}$ (Eq. $2 \mathrm{c}$ ).

$$
V_{\text {total }}=V_{B B}+V_{\text {Shadow }}
$$

with

$$
\begin{aligned}
& V_{B B}=\sum V_{B B, i} \\
& V_{B B, i}=\left(X_{\max , i}-X_{\min , i}\right) \cdot\left(Y_{\max , i}-Y_{\min , i}\right) \cdot s_{\text {voxel }} \\
& V_{\text {Shadow }}=n_{O} \cdot\left(s_{\text {voxel }}{ }^{3}\right)
\end{aligned}
$$

$$
\begin{array}{ll}
\text { where: } & \\
V_{B B} & \ldots \text { sum of all bounding box volumes } \\
V_{B B, i} & \ldots \text { individual bounding box volume for voxel } c_{i} \\
V_{\text {Shadow }} & \ldots \text { volume of all occluded voxel } \\
S_{\text {voxel }} & \ldots \text { unit voxel volume } \\
n_{o} & \ldots \text { number of occluded voxels }
\end{array}
$$

\subsection{Reference volume estimation via xylometry methods}

The direct volume measurement (in the following referred to as $V_{R e f}$ ) was performed by cutting the tree into pieces and placing the branch and trunk components into a water tank (Figure 5). The displaced water ran off via an overflow pipe and was collected. By measuring the weight of the collected water, $V_{R e f}$ was determined. This method is very precise because trunk and branch discontinuities (knots, grooves etc.) are considered.

For both trees (T2 and T6), the reference volume $V_{\text {Ref }}$ was obtained separately for the trunk, the branches and the complete tree. 


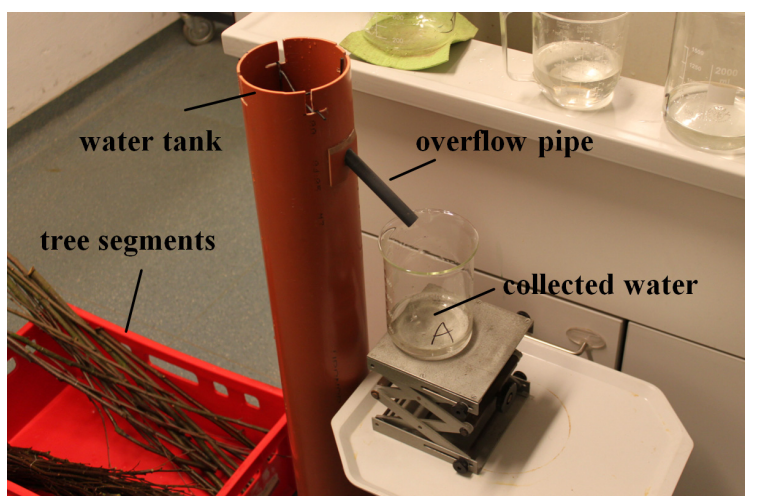

Figure 5. Experimental set-up. (C. Hess, Leuphana University Lüneburg)

\subsection{Volume estimation via frustum segments}

For the reference volume estimation via frustum segments (Figure 6), diameter measurements along the trunk were taken with a calliper at $20 \mathrm{~cm}$ intervals. The trunk is represented by a number of frustums with a height $h_{f}=20 \mathrm{~cm}$. Using the radius of the bottom area $R_{l}$ and top area $R_{2}$ of the frustum, the volume $V_{F r u}$ is determined (Eq. 3). The frustum modelling comprises only the trunk without branches.

$V_{F r u}=h_{f} \cdot \pi / 3 \cdot\left(R_{1}^{2}+R_{1} R_{2}+R_{2}^{2}\right)$

Eq. 3

where:

$$
\begin{array}{lll}
h_{f} & \ldots \text { frustum height } \\
R_{1} & \ldots \text { radius of bottom area } \\
R_{2} & \ldots \text { radius of top area }
\end{array}
$$

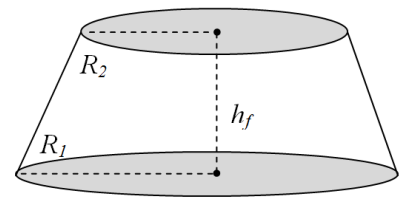

Figure 6. Frustum of a cone.

\section{RESULTS}

A first test was performed with different voxel sizes. Figure 7 shows the steps of voxel generalisation using the example of tree T3 under leaf-off conditions. Due to the size of the stem diameter, a small voxel size (i.e., $0.5 \mathrm{~cm}$ or $1 \mathrm{~cm}$ ) visually suits best to represent the tree volume.

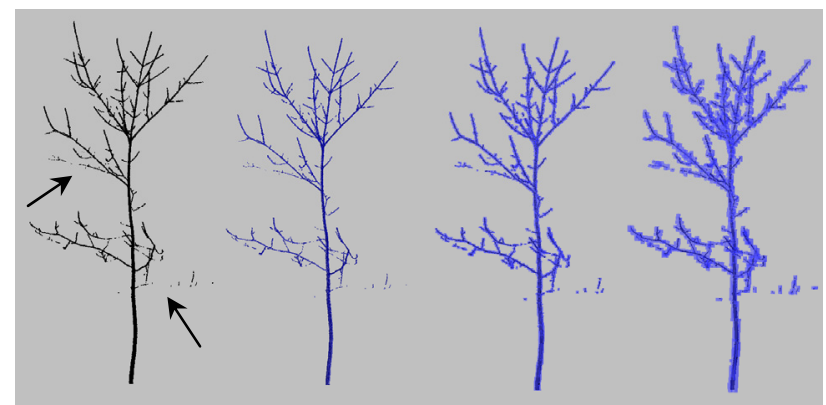

Figure 7. Steps of generalisation with missing laser scanner points due to filtering. From left to right: Point cloud of a tree; voxel spaces overlaid with point cloud (voxel size: $0.5 \mathrm{~cm}, 2 \mathrm{~cm}, 4 \mathrm{~cm}$ ).
The results of the analysed trunks of data set A are shown in Table 1. The table lists $h, d b h$ and the four volume determinations ( $\left.V_{R e f}, V_{F r u}, V_{V o x}, V_{\text {total }}\right)$ for all scanned trees with the minimum and maximum differences and a root mean squared error (RMSE) compared to the reference volume. The deviation $\Delta V_{\%}=V_{\text {Method }}-V_{R e f}$ is also depicted. It was found that $V_{F r u}$ matches $V_{\text {Ref }}$ well with slight over- and underestimations ranging from $-9.1 \%$ to $11.0 \%$ $(\mathrm{RMSE}=6.4 \%)$.

As expected, an extreme overestimation (47\% up to $383 \%$ ) was obtained for the $V_{V o x}$ with a small voxel size of $s_{\text {voxel }}=1 \mathrm{~cm}$. $\Delta V_{\%}$ strongly increases with decreasing $d b h$. The volume $V_{V o x}$ of the trunks are always overestimated, even when occluded inner stem voxels are not considered.

A more accurate volume determination was determined by using the expanded bounding boxes and occluded voxel volumes compared to the reference volume ( $\Delta V_{\%}$ from $-17.7 \%$ up to $22.0 \%$ with a RMSE of $11.6 \%$ ). With an approximately normal distribution of the deviations, the sum of the $V_{\text {total }}$ of all 13 trunks deviates from $V_{\text {Ref }}$ by only $-0.3 \%$.

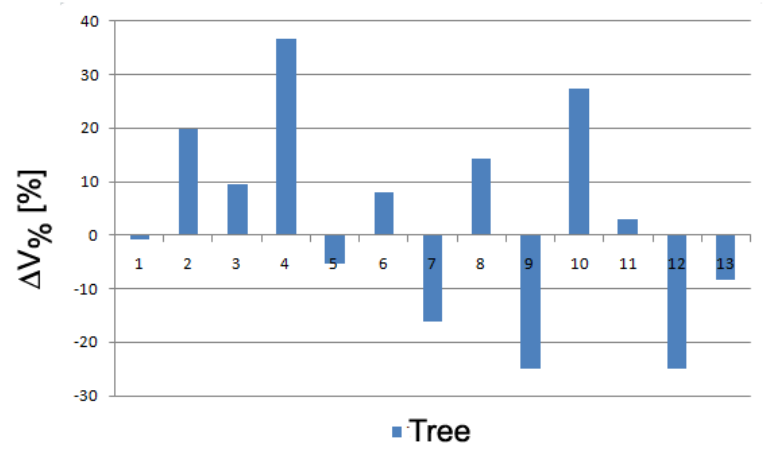

Figure 8. Percentage deviations $\Delta V_{\%}$ of the trees $\Delta V_{\%}=100 \cdot\left(V_{\text {total }}-V_{\text {Ref }}\right) / V_{\text {Ref }}$.

Figure 8 presents the results of the volume determination of the complete tree point clouds compared to $V_{R e f}$. The error of the sum of the total volume of all trees, obtained by summing the volumes $V_{\text {total }}$ of each tree and compared with the sum $V_{\text {Ref }}$ of all trees, is $6.8 \%$ with a RMSE of $18.5 \%$.

The results of the direct volume measurements (via xylometry and via calliper measurements) for data set B are depicted in Table 2. It is shown, that $V_{F r u}$ of the trunk $\mathrm{T} 2$ is slightly overestimated $(4.3 \%)$ and the trunk $\mathrm{T} 6$ is underestimated $(-9.1 \%)$ in comparison with $V_{R e f}$.

\begin{tabular}{llcc}
\hline Method & Volume & $\begin{array}{c}\text { Data set B1 } \\
\text { Tree T2 } \\
{\left[\mathbf{m}^{3}\right]}\end{array}$ & $\begin{array}{c}\text { Data set B2 } \\
\text { Tree T6 } \\
{\left[\mathbf{m}^{3}\right]}\end{array}$ \\
\hline $\boldsymbol{V}_{\text {Fru }}$ & Trunk & 0.003953 & 0.000140 \\
$\boldsymbol{V}_{\text {Ref }}$ & Trunk & 0.003789 & 0.000154 \\
$\boldsymbol{V}_{\text {Ref }}$ & Branches & 0.001854 & 0.000018 \\
$\boldsymbol{V}_{\text {Ref }}$ & Tree & 0.005643 & 0.000172 \\
\hline
\end{tabular}

Table 2. Direct volume measurements of data set $\mathrm{B}$; $V_{\text {Ref }}=$ volume determination via xylometry, $V_{F r u}=$ volume determination via frustum modelling. 


\begin{tabular}{|c|c|c|c|c|c|c|c|c|c|}
\hline \multirow[t]{2}{*}{ Trunk } & \multirow{2}{*}{$\begin{array}{c}\begin{array}{c}\text { Height } \\
(h)\end{array} \\
{[\mathrm{m}]} \\
\end{array}$} & \multirow{2}{*}{$\begin{array}{c}\begin{array}{c}\text { Diameter } \\
\text { at breast } \\
\text { height } \\
(\text { dbh })\end{array} \\
{[\mathrm{m}]} \\
\end{array}$} & \multirow{2}{*}{$\begin{array}{c}\begin{array}{c}\text { Reference } \\
\text { volume } \\
\left(V_{\text {Ref }}\right)\end{array} \\
{\left[\mathrm{m}^{3}\right]}\end{array}$} & \multicolumn{2}{|c|}{$\begin{array}{l}\text { Frustum segments } \\
\left(V_{F r u}\right)\end{array}$} & \multicolumn{2}{|c|}{$\begin{array}{c}\text { Voxel volume method } \\
\left(V_{\text {Vox }}, s_{\text {voxel }}=1 \mathrm{~cm}\right)\end{array}$} & \multicolumn{2}{|c|}{$\begin{array}{l}\text { Bounding box and } \\
\text { occluded voxel volume } \\
\text { method }\left(V_{\text {total }}\right)\end{array}$} \\
\hline & & & & {$\left[\mathbf{m}^{3}\right]$} & $\Delta V_{\%}$ & {$\left[\mathbf{m}^{3}\right]$} & $\Delta V_{\%}$ & {$\left[\mathbf{m}^{3}\right]$} & $\Delta V_{\%}$ \\
\hline$T 1$ & 3.42 & 0.028 & 0.002233 & 0.002257 & 1.1 & 0.003368 & 50.8 & 0.002140 & -4.2 \\
\hline$T 2$ & 3.98 & 0.041 & 0.003789 & 0.003953 & 4.3 & 0.005059 & 33.5 & 0.003783 & -0.1 \\
\hline$T 3$ & 4.87 & 0.041 & 0.004097 & 0.004170 & 1.8 & 0.005604 & 36.8 & 0.004192 & 2.3 \\
\hline$T 4$ & 3.33 & 0.023 & 0.001296 & 0.001217 & -6.1 & 0.003113 & 140.2 & 0.001582 & 22.0 \\
\hline$T 5$ & 3.12 & 0.018 & 0.000875 & 0.000840 & -4.0 & 0.002003 & 128.9 & 0.000738 & -15.7 \\
\hline$T 6$ & 1.92 & 0.008 & 0.000154 & 0.000140 & -9.1 & 0.000645 & 318.8 & 0.000162 & -5.3 \\
\hline$T 7$ & 2.18 & 0.012 & 0.000243 & 0.000232 & -4.5 & 0.000744 & 206.2 & 0.000200 & -17.7 \\
\hline$T 8$ & 1.79 & 0.011 & 0.000283 & 0.000260 & -8.1 & 0.001025 & 262.2 & 0.000323 & 14.1 \\
\hline$T 9$ & 2.23 & 0.014 & 0.000408 & 0.000380 & -6.9 & 0.001148 & 181.4 & 0.000360 & -11.7 \\
\hline$T 10^{*}$ & 4.75 & 0.028 & 0.002300 & 0.002127 & -7.5 & 0.005301 & 130.5 & 0.002650 & 15.2 \\
\hline$T 11$ & 4.88 & 0.020 & 0.001335 & 0.001235 & -7.5 & 0.003355 & 151.3 & 0.001282 & -4.0 \\
\hline$T 12$ & 1.49 & 0.030 & 0.001439 & 0.001597 & 11.0 & 0.002190 & 52.2 & 0.001486 & 3.3 \\
\hline$T 13$ & 1.45 & 0.028 & 0.000730 & 0.000750 & 2.7 & 0.001389 & 90.3 & 0.000672 & -8.0 \\
\hline Sum & - & - & 0.023125 & 0.023251 & 0.5 & 0.040273 & 74.2 & 0.023197 & -0.3 \\
\hline Min & 1.45 & 0.008 & & & -9.1 & & 33.5 & & -17.7 \\
\hline $\operatorname{Max}$ & 4.88 & 0.041 & & & 11.0 & & 318.8 & & 22.0 \\
\hline RMSE & & & & & 6.4 & & 161.2 & & 11.6 \\
\hline
\end{tabular}

* due to tree movement the tree structure was mapped twice

Table 1. Volume determinations of the trunks of data set A via frustum segments $\left(V_{F r u}\right)$, voxels $\left(V_{V o x}\right)$ and bounding boxes with occluded voxels $\left(V_{\text {total }}\right)$ of the trunks of young Norway maple trees compared with the reference measurements $\left(V_{R e f}\right)$.

The following tests are divided in volume analyses regarding the different tree components (trunk, branches, complete tree) separately to assess the quality of the results. Table 3 presents the percentage volume differences $\left(\Delta V_{\%}=V_{\text {total }}-V_{R e f}\right)$ of the trees $\mathrm{T} 2$ and $\mathrm{T} 6$ for two scan epochs. The volume determination of the trunks shows high congruence between the two scan epochs. In contrast, the volume determinations of the branches yields large deviations. In general, an overestimation would be expected, but the two trees have very thin branches, and some of the laser scanning points were eliminated in the point cloud filtering to eliminate the scanner specific stray points (see Figure 7, arrows). This results in an underestimation of the volume. The determination of the total tree volume yields differences of $-12 \%$ up to $20 \%$ for T 2 and $-12 \%$ up to $8 \%$ for T6. It has to be noted that $V_{\text {total }}$ of the tree was obtained by processing the whole point cloud, i.e., it is not the sum of the trunk and the branch volume. Summarizing, the test showed a repeatability of about $10 \%$ for wood volume.

\begin{tabular}{llcccc}
\hline \multirow{2}{*}{ Tree parts } & $\begin{array}{l}\text { Volume } \\
\text { differences }\end{array}$ & $\begin{array}{c}\text { T2_a } \\
{[\%]}\end{array}$ & $\begin{array}{c}\text { T2_b } \\
{[\%]}\end{array}$ & $\begin{array}{c}\text { T6_a } \\
{[\%]}\end{array}$ & $\begin{array}{c}\text { T6_b } \\
{[\%]}\end{array}$ \\
\hline Trunk & $V_{\text {total }}-V_{\text {Ref }}$ & -0.1 & -8.1 & 5.3 & -5.5 \\
Branches & $\boldsymbol{V}_{\text {total }}-V_{\text {Ref }}$ & 70.1 & -21.5 & -61.1 & -80.2 \\
Tree & $V_{\text {total }}-V_{\text {Ref }}$ & 19.8 & -12.5 & 8.0 & -12.6 \\
\hline
\end{tabular}

Table 3. Volume differences of data set B for $V_{\text {total }}-V_{\text {Ref }}$ split into trunk, branches and tree. a indicates scan epoch 1 and_b indicates scan epoch 2.
Another test was performed to show the dependency of the results on the orientation of the voxel structure in the point cloud. Using an axis-parallel bounding box to determine the contour of a point cloud slice does not always present the minimum volume depending on the orientation of the points inside the coordinate system. To estimate the effect of this, a stepwise rotation $\left(\Delta \alpha=15^{\circ}\right)$ of the point cloud around the $Z$ axis was performed to simulate different point distributions inside a voxel and its obtained volumes. The average $\Delta \overline{V_{\%}}$ (Eq. 4) of all rotated volumes $n$ (23 per tree) to the initial volume $\left(\alpha=0^{\circ}\right)$ was calculated. The RMSE, the minimum and maximum volume differences are shown for each trunk and tree in Table 4.

$$
\begin{gathered}
\Delta \overline{V_{\%}}=\frac{\sum_{\alpha=15^{\circ}}^{345^{\circ}}\left(V_{\text {total }}^{\alpha}-V_{\text {total }}^{0}\right)}{n} \\
\text { with } \quad \begin{array}{l}
V_{\text {total }}^{0}=\text { initial volume with } \alpha=0^{\circ} \\
n=23 \\
\Delta \alpha=15^{\circ}
\end{array}
\end{gathered}
$$

As shown in Table 4, the influence of the orientation of the scan data show average volume values with a maximum RMSE of $8.5 \%$ for trunk T9 and $7.4 \%$ for tree T9. Analysing the minimum and maximum deviations show that the determined volumes vary up to $13 \%$ (disregarding tree T9) . The analysis shows a dependency on the data set orientation, which is recognizable, but significantly smaller than the overall accuracy of the method. 


\begin{tabular}{|c|c|c|c|c|c|c|}
\hline \multirow[b]{2}{*}{ No } & \multicolumn{3}{|c|}{ Segmented trunk } & \multicolumn{3}{|c|}{ Complete tree } \\
\hline & $\begin{array}{c}\text { RMSE } \\
{[\%]}\end{array}$ & $\begin{array}{c}\Delta_{\min } \\
{[\%]}\end{array}$ & $\begin{array}{c}\Delta_{\max } \\
{[\%]}\end{array}$ & $\begin{array}{c}\text { RMSE } \\
{[\%]}\end{array}$ & $\begin{array}{c}\Delta_{\min } \\
{[\%]}\end{array}$ & $\begin{array}{l}\Delta_{\max } \\
{[\%]}\end{array}$ \\
\hline T1 & 1.5 & -2.8 & 1.2 & 2.9 & -4.0 & 0.0 \\
\hline T2 & 0.6 & -0.7 & 0.8 & 0.6 & -0.8 & 0.5 \\
\hline T3 & 0.9 & -1.8 & 0.5 & 1.1 & -1.9 & 0.5 \\
\hline T4 & 4.6 & -6.5 & 0.9 & 4.4 & -6.4 & 1.0 \\
\hline T5 & 4.5 & -7.8 & 3.3 & 3.7 & -6.0 & 2.5 \\
\hline T6 & 3.6 & -3.2 & 5.0 & 4.1 & -3.1 & 6.1 \\
\hline T7 & 5.8 & -9.5 & 2.5 & 5.7 & -9.0 & 2.3 \\
\hline T8 & 5.6 & -7.4 & 0.1 & 5.2 & -7.0 & 1.0 \\
\hline T9 & 8.5 & -12.7 & 4.4 & 7.4 & -10.9 & 3.3 \\
\hline T10 & 3.0 & -3.7 & 0.9 & 3.1 & -4.1 & 0.7 \\
\hline T11 & 3.5 & -4.7 & 4.0 & 4.1 & -5.4 & 3.5 \\
\hline T12 & 1.3 & -1.1 & 2.1 & 1.4 & -2.0 & 0.9 \\
\hline T13 & 5.0 & -9.6 & 4.1 & 5.0 & -9.6 & 4.1 \\
\hline
\end{tabular}

Table 4. RMSE and the minimum and maximum deviations of the average rotated volume differences to the initial volume $\left(\alpha=0^{\circ}\right)$ per trunk and tree.

\section{DISCUSSION}

This non-destructive method allows for an automatic volume determination of wooden parts of a tree. It is an objective technique without errors made by humans during measuring. As stated in Hosoi et al. (2013), a major advantage can be seen in the fact that the stem shape is not approximated by symmetrical bodies, allowing to take account of the real stem shapes with irregularities.

For identifying the occluded voxels accurately, the method needs closed contours of stems and branches as input data. That means that a complete $360^{\circ}$ scan of the tree has to be captured, i.e. multiple scans and a data registration have to be performed. At least at one position the scanner has to be levelled during recording. Concerning the weather conditions, it has to be windless (always necessary when scanning in forests). Preferably, the trees should be recorded under leaf-off conditions to avoid occlusion by the leaves. In leaf-on conditions there will be a total loss of information in scanning especially of fine branches due to massive occlusion by leaves.

The results in Table 3 demonstrate a good repeatability of the method of about $10 \%$ for the trunk volumes. After separating the point cloud in trunk and branches, holes remain at the location of the branch. This results in an underestimation because of lacking wood structure. A variable object size results in different voxel spaces (voxel space definition via bounding box of the complete point cloud) with different filled voxels and volumes. That is a reason why the volume of the processed tree point cloud differs from to the sum of the partially processing (Table 3). Nevertheless, a sample set of only two point clouds per tree for testing the repeatability is very definitive. The number has to be enlarged in future studies. It was shown, that the orientation of the point cloud influences the volume determination. Maybe a transformed bounding box instead of an axis-parallel orientation, along the principal components suits better for the volume results of the outer voxels. Also the scan registration affects the accuracy. Open stem contours, due to registration errors, may affect the number of the occluded voxels.

Finally, the results show a good agreement with the total wood volume, despite that some candidates show large deviations. It has to be noted that a very precise method was chosen as reference. When comparing the method with conventional methods (calliper measurements, taper equations), which also show deviations from the reference, the results become even better. Overall, the method is suitable for a relative volume analysis of wooden structure to determine the vegetation growth over several years. Thus, the error has not much impact during the usage of volume differences.

\section{SUMMARY}

The paper presents an algorithm to estimate the wood volume of trees using a voxel-based method which avoids overestimation of the volume. The method works with a (filtered) tree point cloud in a horizontal coordinate system. The results show good agreements with the reference measurements via xylometry methods. A sum of all trunk volumes of $-0.3 \%$ with a RMSE of $11.6 \%$ indicates a slight underestimation of the method. In general, the results are influenced by the scan registration, the tree structure and tree orientation. Under good scan conditions, the laser scanner derived volume accuracy is comparable with frustum segments measurements, which are established in forestry. Future work should evaluate the potential of alpha shapes or other 3D convex hull techniques applied to the segmented point clouds. Practical tests will also apply the method to other tree species, tree sizes and voxel sizes.

\section{REFERENCES}

Dassot, M.; Colin, A.; Santenoise, P.; Fournier, M. \& Constant, T., 2012. Terrestrial laser scanning for measuring the solid wood volume, including branches, of adult standing trees in the forest environment. Computers and Electronics in Agriculture, 89, pp. 86-93.

Hosoi, F.; Nakai, Y. \& Omasa, K., 2013. Voxel tree modeling for estimating leaf area density and woody material volume using 3-D lidar data. In: IAPRS, Antalya, Turkey, Vol. II-5/W2, pp. 115-120.

Hopkinson, C.; Chasmer, L.; Young-Pow, C. \& Treitz, P., 2004. Assessing forest metrics with a ground-based scanning lidar. Canadian Journal of Forest Research, 34, pp. 573-583.

Kankare, V.; Holopainen, M.; Vastaranta, M.; Puttonen, E.; Yu, X.; Hyyppä, J.; Vaaja, M.; Hyyppä, H. \& Alho, P., 2012. Individual tree biomass estimation using terrestrial laser scanning. ISPRS Journal of Photogrammetry and Remote Sensing, 75, pp. 64-75.

Li, Y.; Hess, C.; von Wehrden, H.; Härdtle, W. \& von Oheimb, G., 2014. Assessing tree dendrometrics in young regenerating plantations using terrestrial laser scanning. Annals of Forest Science, INRA and Springer-Verlag France 2014.

Maas, H.-G.; Bienert, A.; Scheller, S. \& Keane, E., 2008. Automatic forest inventory parameter determination from terrestrial laserscanner data. International Journal of Remote Sensing, 29(5), pp. 1579-1593.

Moskal, M. \& Zheng, G., 2011. Retrieving Forest Inventory Variables with Terrestrial Laser Scanning (TLS) in Urban Heterogeneous Forest. Remote Sensing, 2012, 4, pp. 1-20.

Pueschel, P.; Newnham, G.; Rock, G.; Udelhoven, T.; Werner, W. \& Hill, J., 2013. The influence of scan mode and circle fitting on tree stem detection, stem diameter and volume extraction from terrestrial laser scans. ISPRS Journal of Photogrammetry and Remote Sensing, 77, pp. 44-56.

Thies, M. \& Spiecker, H., 2004. Evaluation and future prospects of terrestrial laser scanning for standardized forest inventories. In: IAPRS, Freiburg, Germany, Vol. 36, Part 8/W2, pp.192-197.

Watt, P.J.; Donoghue, D.N.M. \& Dunford, R.W., 2005. Measuring forest structure with terrestrial laser scanning. International Journal of Remote Sensing, 26(7), pp. 1437-1446 